\title{
A structure-based argument for non-classical crystal growth in natural clay minerals
}

\author{
Emilia García-Romero ${ }^{1,2, *}$ and Mercedes Sú́rez ${ }^{3}$ \\ 1 Department of Crystallography and Mineralogy, Faculty of Geology, Complutense University of Madrid, C/ José \\ Antonio Nováis 12, 28040 Madrid, Spain. \\ 2 Instituto de Geociencias (IGEO), Complutense University of Madrid and Consejo Superior de Investigaciones \\ Científicas, C/ José Antonio Nováis 12, 28040 Madrid, Spain. \\ 3 Department of Geology, University of Salamanca, Plaza de la Merced s/n. 37008 Salamanca, Spain.
}

[Received 15 December 2016; Accepted 14 May 2017; Associate Editor: Andrew Christy]

\section{ABSTRACT}

Evidence of crystallization by particle attachment in synthetic materials is described in numerous contributions. However, efforts to establish the contribution of the particle attachment mechanism to inorganic crystallization in natural environments have barely begun. Here, we show, for the first time, evidence that confirms oriented particle attachment as a crystal growth mechanism that is relevant in sedimentary environments. In these natural settings, oriented particle attachment operates during the formation of highly anisotropically structured clay minerals, which constitute one of the most extensively distributed groups of minerals in the Earth's crust. High-resolution transmission electron microscopy images show that the clay minerals aggregation process occurs in different manners. Smectites aggregate by semi-oriented attachment, while kaolinite, sepiolite and palygorskite aggregate by oriented attachment.

KEYWORDS: crystal growth, sepiolite, palygorskite, kaolinite, clay minerals, oriented attachment, mesocrystal, aggregation.

\section{Introduction}

SinCE the late 1990s, when the first studies on oriented attachment (OA) of crystals were published (Penn and Banfield, 1998; 1999; Banfield et al., 2000), this non-classical mechanism of crystal formation has been researched extensively. Although numerous studies have been conducted previously (Ivanov et al., 2014), non-classical crystallization has only been recognized widely in the past few years. Evidence of crystallization in biogenic and synthetic materials (e.g. metal oxides, selenides and sulfides) by particle attachment is demonstrated in numerous contributions, and this process has even been observed directly by high-resolution transmission electron microscopy (HRTEM) using a fluid cell (Li et al., 2012).

*E-mail: mromero@ucm.es

https://doi.org/10.1180/minmag.2017.081.031
However, efforts to establish the contribution of the particle attachment mechanism to inorganic crystallization in natural environments have barely begun (De Yoreo et al., 2015). In this work, we present for the first time evidence that confirms oriented particle attachment as a crystal growth mechanism that is relevant in sedimentary environments. In these natural settings, oriented particle attachment operates during the formation of the structurally highly anisotropic clay minerals, which constitute one of the most extensively distributed groups of minerals in the Earth's crust.

The classical theory of crystallization assumes the incorporation of individual ions or molecules onto high-energy crystal faces. However, after the pioneering work of Penn and Banfield (1998), today, it is generally accepted that crystals can also grow by the oriented addition of clusters, nanoparticles, or even micrometric crystals (Viedma et al., 2013). The formation of (single) crystals through oriented self-assembly into superstructures, followed 
by later fusing of nanoparticles originated in parallel multiple nucleation events, is included within the category of 'nonclassical crystallization'. Oriented attachment generates mesocrystals that are defined as kinetically stabilized superstructures of nanocrystals arranged in crystallographic co-alignment (Penn, 2004) in which the primary units can still be identified. Therefore, mesocrystals are an intermediate step between dispersed particles and true single crystals (Colfen and Antonietti, 2008). The first references to crystallization by OA were related mainly to $\mathrm{Fe}$, $\mathrm{Ti}$ or $\mathrm{Zn}$ synthetic oxides (Penn, and Banfield, 1998; 1999; Penn et al, 2001, Niederberger, et al., 2002, Cozzoli et al., 2003) and other synthetic compounds, such as sulfides (Zhang, 2000; Ricolleau, 1999) or zeolites (Nikolakis et al., 2000). After these studies, hundreds of references have described OA for a diverse range of synthetic compounds. Oriented attachment has often been argued as the mechanism responsible for the formation of natural biogenic iron-oxide nanoparticles (Banfield et al., 2000). In more recent experimental studies, OA has been proposed as a possible mechanism for the formation of todorokite in deep-sea polymetallic nodules (Atkins et al., 2014). Oriented attachment has even been proposed for the growth of crystals not only from solutions but also from melts (Helmy et al., 2013). Consequently, OA has been used to explain the existence of platinum-group elements in pentlandite (Junge et al., 2015) but has never been demonstrated. In a previous work, we have shown evidence of mesocrystals of sepiolite and palygorskite (García-Romero and Suárez, 2014).

Clay minerals are ubiquitous in the Earth's surface environments, where they form mainly by hydrolysis from less stable silicates and by direct precipitation from solutions. Apart from their importance as rock-forming minerals and palaeoenvironmental indicators, the properties of clays are highly advantageous in numerous industries. In this study, we provide evidence to support the claim that the growth of clay minerals in sedimentary environments occurs mainly by OA. Furthermore, compared to classical mechanisms, we state that, due to the structural characteristics of clay minerals, $\mathrm{OA}$ is a much more efficient mechanism for their crystal growth, and it is the only mechanism that can account for the development of their specific morphological features. The process has been proven herein using HRTEM images of clay minerals.

\section{Methods}

High-resolution TEM imaging was conducted at the Centro Nacional de Microscopía Electrónica (Spain). Clay minerals are extremely beam-sensitive compared to other non-hydrated minerals, posing a higher level of difficulty for HRTEM imaging. Therefore, samples must be prepared under special conditions to prevent beam damage. The experimental conditions were optimized to avoid structural modification using a low beam intensity ( $<500$ counts on the CCD camera) with an exposure time of $0.8 \mathrm{~s}$ to acquire the image. The samples were prepared via two methods: the conventional method of depositing a drop of dilute clay suspension onto a microscope $\mathrm{Cu}$ grid with cellulose acetate butyrate; and by studying ultrathin sections of undisturbed samples. The samples underwent treatments to preserve the microtexture and avoid the collapse of the smectite interlayer space. These treatments are conducted in a sequence of successive steps where a small portion of sample is placed in agar-agar to protect it from future stains. The sample must then be hydrated and the water progressively replaced by alcohol; afterwards the alcohol is replaced by Spurr resin, according to the methodology proposed by Tessier (1984) and Tessier and Pedro (1987). After polymerization of the resin, thin sections $(50 \mathrm{~nm})$ were cut by ultramicrotomy. This procedure minimizes dehydration during HRTEM study and thus helps preserve the natural texture of the sample. The observations were performed using a JEOL 3000 FX field-emission microscope with $\mathrm{LaB}_{6}$ filament at an acceleration voltage of $300 \mathrm{kV}$ with $0.17 \mathrm{~nm}$ point-to-point resolution, equipped with a doubletilt sample holder (up to a maximum of $\pm 23^{\circ}$ ) and a CCD camera for digitally recording the images. The images shown herein have been selected from the authors' photo collections. The samples come from different localities around the world that have been studied previously by the authors, with different objectives.

\section{Evidence of oriented aggregation in clay minerals by HRTEM observations}

The HRTEM images of different clay minerals (kaolinite, smectite, and sepiolite-palygorskite) indicate that all are composed of small crystallites. Close inspection reveals that the crystallites are in turn composed of smaller subunits, and that 
structural and textural peculiarities of the different clay minerals are related to the arrangement of these subunits. Figure 1 has been obtained from the sedimentation of kaolinite particles on the grid. In these images, kaolinite appears as pseudohexagonal plates of different sizes. The smaller particles that appear overlapped mostly have the same crystallographic orientation (indicated as $\mathrm{OA}$ in Fig. 1), and they can be distinguished perfectly from the misoriented overlapping particles (indicated as $\mathrm{OV}$ in Fig. 1c). The oriented overlapping indicates the aggregation of individual units forming larger particles, while the misoriented overlapping is the result of sedimentation of different particles on the grid that have, in this case, different orientations. The size of the crystals that aggregate to form the kaolinite mesocrystals ranges from $50 \mathrm{~nm}$ to $500 \mathrm{~nm}$.

High-resolution TEM images of the smectite show that this clay mineral occurs as lenticular packets with elongate sigmoidally curved crosssections (Fig. 2 and 3 ) and tapered margins. These particles are usually no larger than $1 \mu \mathrm{m}$ long by $50 \mathrm{~nm}$ wide at their central part. Under higher magnification, lattice planes with a spacing of $14 \AA$ become visible with numerous stacking faults and edge dislocations. The stacking defects concentrate near particle edges (Figs 2 and 3). This lattice spacing matches that expected from the stacking of smectite structural units (the 2:1 layers) along the c-axis direction. It is interesting to note that smectite particles also usually consist of smaller, similar individual particles (like smaller sigmoid) (Figs 2 and 3), indicating that they originated by the addition of minor single particles. The shapes of these particles are a direct consequence of their mode of attachment. In the smectites, the aggregation of the minor single particles (or even isolated layers) that forms the larger particles occurs in a staggered manner by semi-oriented attachment, generating multiple stacking faults and dislocations, as observed in Figs 2 and 3, as is usual for this type of attachment process (De Yoreo et al., 2015). Dislocations and line defects are frequently found next to the interface between the adjacent oriented particles as a consequence of the fit between them, as described by Banfield et al. (2000). In some cases, the interface between the two units is still distinguishable, as in Fig. 4, which shows a detail of the interface between two laminar particles of illite in which its constituent primary units can be distinguished.

In the same way, HRTEM images of natural sepiolite and palygorskite samples show that fibres, irrespective of length are composed of finer-width subunits (Fig. 4) (García-Romero and Suárez, 2014). The length of the fibres varies from $<1 \mu \mathrm{m}$ to centimetres (in some instances) with no gap. The smaller and primary units, named laths, are prismatic and elongated in the caxis direction, are 10-30 $\mathrm{nm}$ in width (Fig. 5), and are parallelogram-shaped in cross-section. These images also indicate that after nucleation,
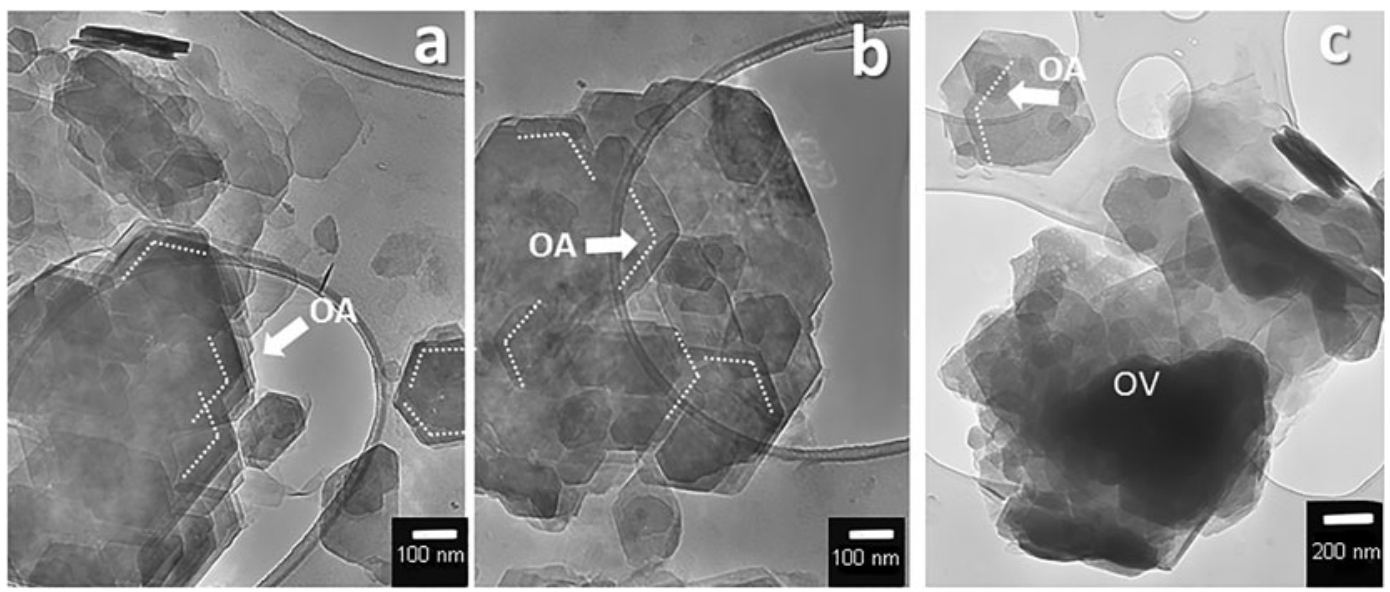

FIG. 1. HRTEM images of pseudohexagonal kaolinite particles obtained by sedimentation on the grid. The smaller size plates are usually overlapped in the same crystallographic orientation on the bigger ones $(a, b)$, while rarely misoriented

(c). OA: Oriented aggregation. OV: overlapped. Samples come from Tamame de Sayago deposit, Zamora, Spain. 


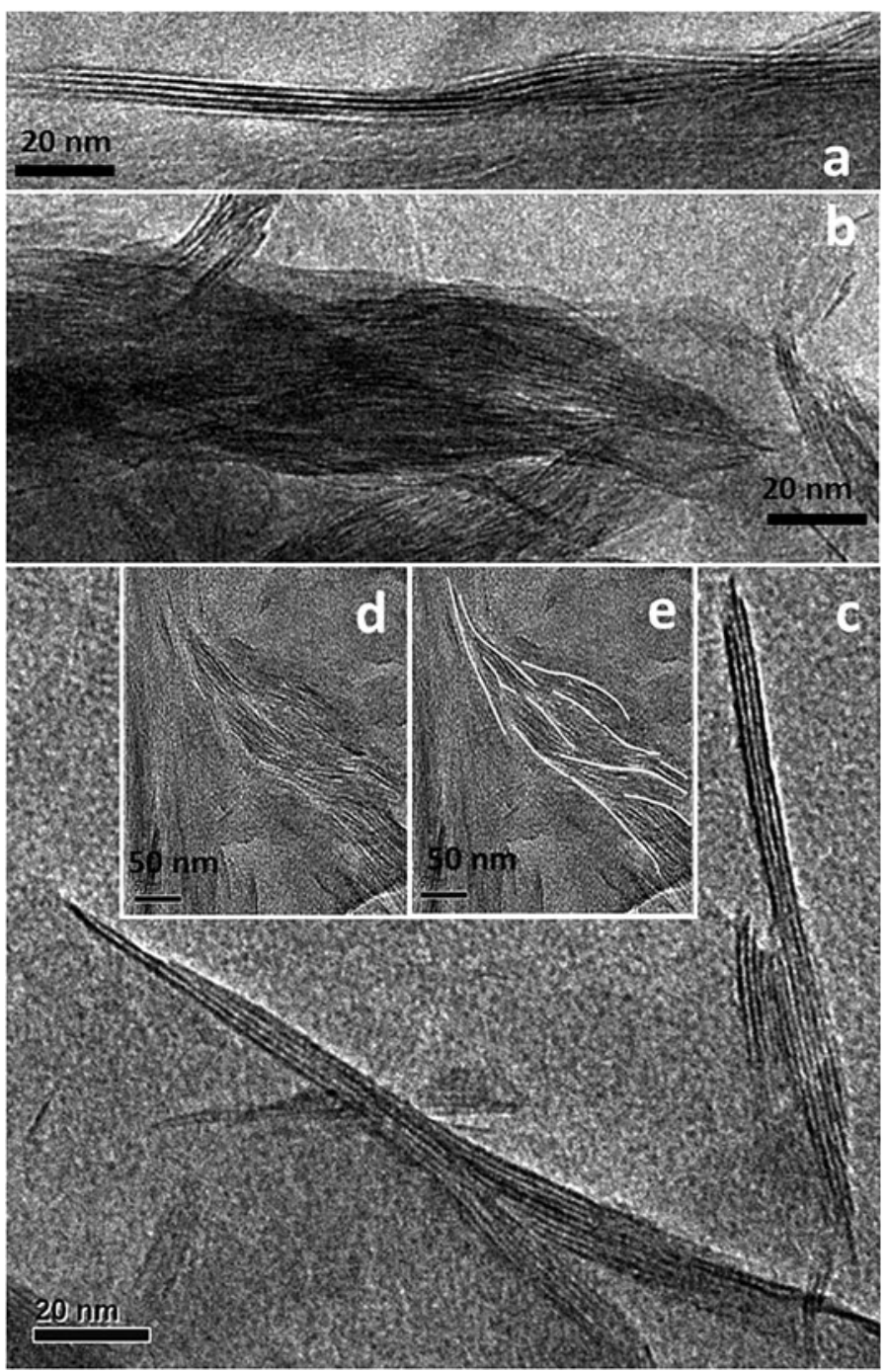

FIG. 2. $(a-e)$ HRTEM smectite images. The particles have a common sigmoidal appearance. Note the parallel lattice planes and the small subunits that form the larger particles. The subunits are thick in their central portions with tapered margins and curved cross-sections. They have numerous stacking faults and edge dislocations. The minor units that compose the particle in $(d)$ are highlighted in $(e)$. Samples $(a)$ and $(c)$ come from Cortijo de Archidona deposit, Almeria, Spain. Sample $(b)$ comes from Esquivias deposits, Tagus Basin, Spain. Samples $(d)$ and $(e)$ come from The Green Clays Unit, Tagus Basin, Spain.

the laths join to form larger units. The TEM images show that the mesocrystals of sepiolite and palygorskite are formed by the attachment of laths, of differing lengths, sharing $(h k 0)$ faces. In fibrous crystals, the aggregation of the smallest units (laths) to generate mesocrystals has also been observed in synthetic todorokite (Atkins et al., 2014).

\section{Structural evidence supporting OA in clay minerals}

After the nucleation stage, the growth of a crystal is controlled by the energy of its binding surfaces, which in turn are controlled by the crystal structure. Clay minerals layers have a highly anisotropic structure built from sheets which stack in the 

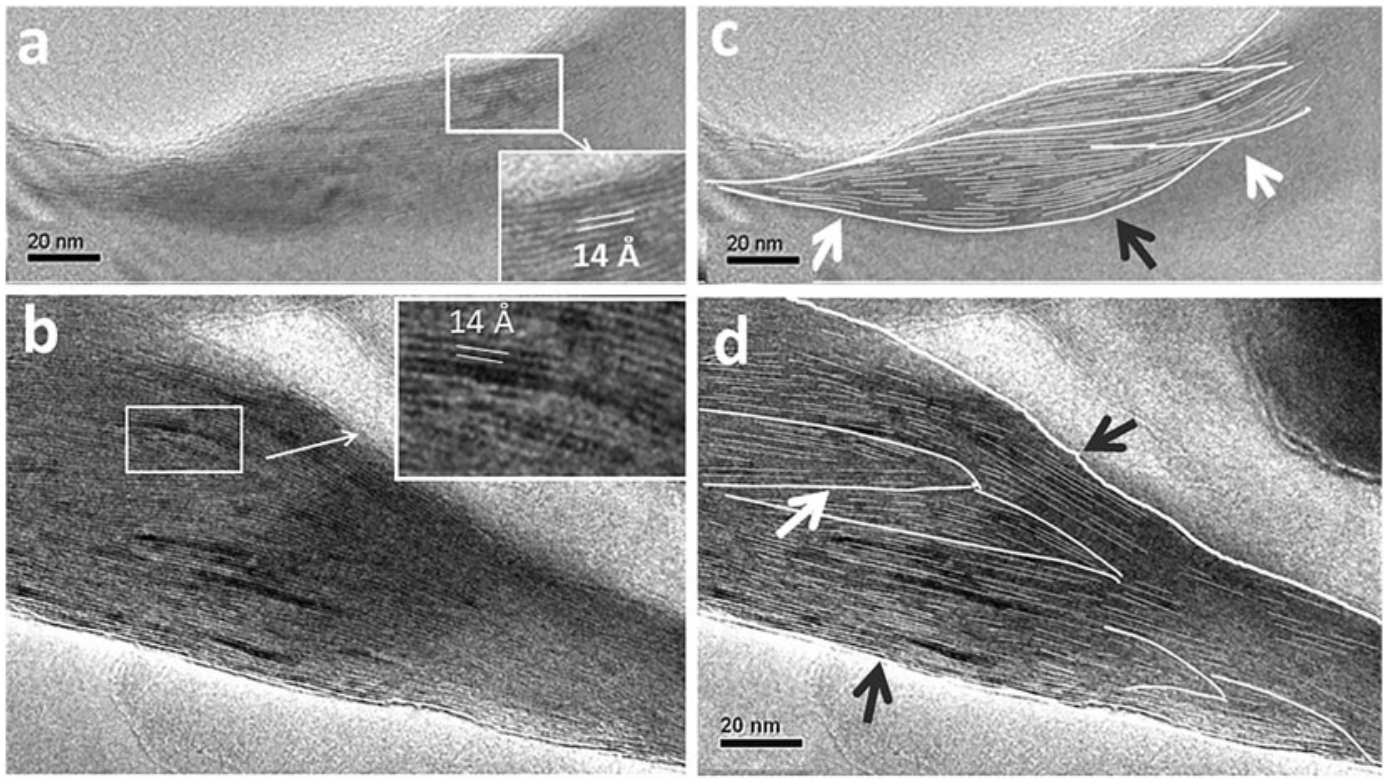

FIG. 3. HRTEM smectite images. Particles formed by the attachment of minor particles. $(a, b)$ Particles of smectite where the parallel lattice planes are observable. They have numerous stacking faults concentrated near the interface of two aggregated particles. The central part of the particles hardly ever has stacking faults. $(c, d)$ The same particles in which the lattice planes, and the units that form them, have been marked. The white arrows indicate areas of higher density of stacking faults. The dark arrows indicate the areas where the parallel lattice planes are free of stacking faults. Samples come from Cortijo de Archidona deposit, Almeria, Spain.

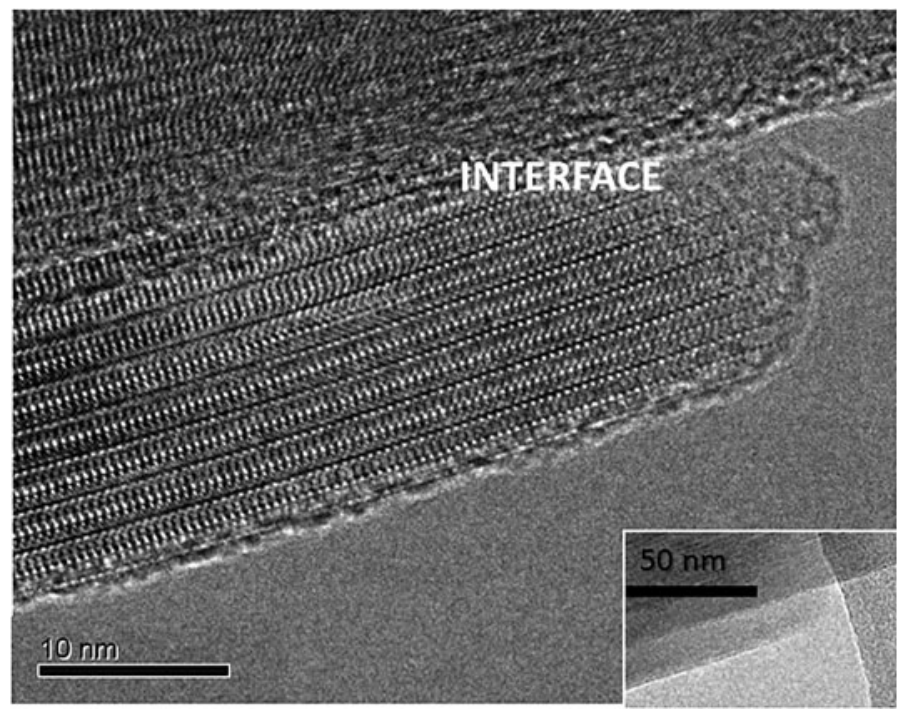

FIG. 4. HRTEM smectite image showing the interface along the union of the two laminar particles (illite). The sample comes from The Green Clays Unit, Tagus Basin, Spain. 

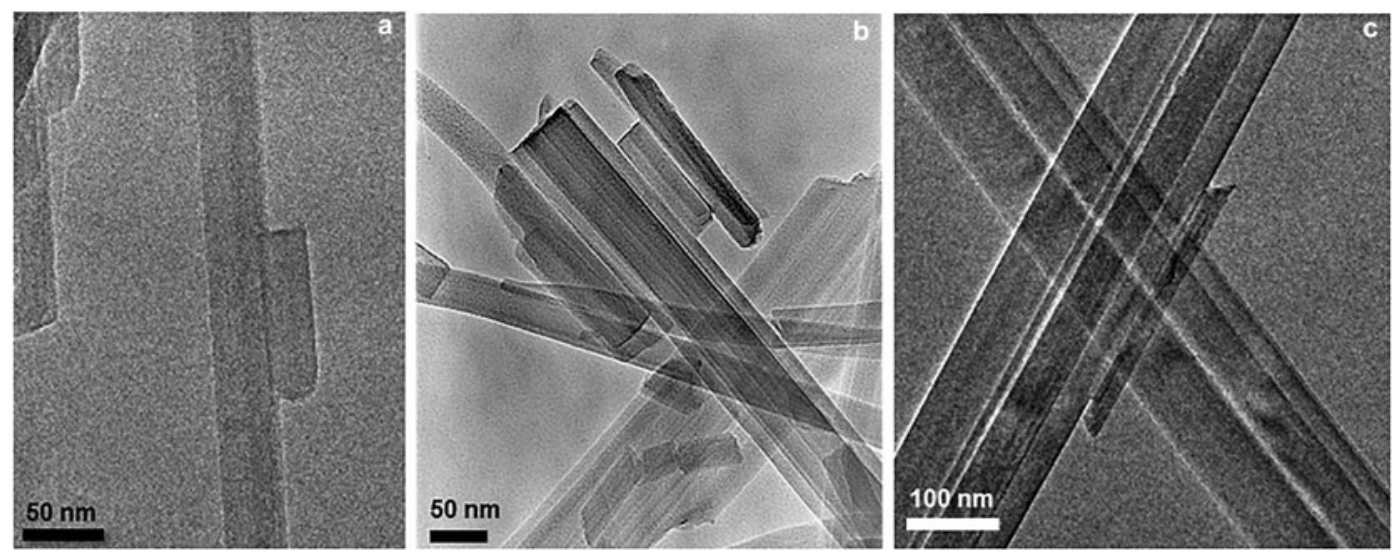

FIG. 5. TEM images of sepiolite-palygorskite sedimented on the grid. The prismatic, elongated particles in the c-axis direction are formed by the attachment of minor ones. Sample $(a)$ comes from the Gran County deposit, New Mexico, USA. Sample $(b)$ comes from the Warren deposit, Texas, USA. Sample $(c)$ comes from the Metaline fault deposit, Washington, USA.

c direction (Figs $6 a$ and $b$ ). Each layer in turn is composed of an octahedral sheet containing larger cations $(\mathrm{Mg}, \mathrm{Al}$ or Fe depending on the mineral species) in octahedral coordination by $\mathrm{OH}^{-}$and $\mathrm{O}^{2-}$, linked to one or two tetrahedral sheets containing $\mathrm{Si}$ in tetrahedral coordination by $\mathrm{O}^{2-}$. Kaolinite crystals are formed by stacked layers composed of one tetrahedral sheet and one octahedral sheet $(\mathrm{T}-\mathrm{O})$ in a 1:1 structure type (Fig. 6a). Kaolinite layers have very few isomorphic substitutions; consequently, they do not have charge or interlayer cations. Additionally, their stacking is due to van der Waals bonds between a face with $\mathrm{OH}^{-}$groups corresponding to an octahedral sheet and another face that is a basal oxygen plane. Smectite crystals are composed of stacked layers formed by one octahedral sheet between two tetrahedral sheets $(\mathrm{T}-\mathrm{O}-\mathrm{T})$ in a $2: 1$ structure type (Fig. 6b). Smectite 2:1 layers have numerous isomorphic substitutions in tetrahedral $\left(\mathrm{Si}^{4+}\right.$ replaced by $\left.\mathrm{Al}^{3+}\right)$ and octahedral positions creating a layer charge, which is compensated by solvated cations in water $\left(\mathrm{Mg}^{2+}, \mathrm{Ca}^{2+}, \mathrm{Na}^{+}\right.$or $\left.\mathrm{K}^{+}\right)$ placed in the interlayer space, named interlayer cations. The weakly charged layers are held together by electrostatic attraction with the cations. Smectite $(001)$ and $(00 \overline{1})$ surfaces are the same because they are formed by the basal oxygen planes of the two tetrahedral sheets and are surrounded by solvated cations. Therefore, for the phyllosilicates, bonding within sheets is strong, while bonding between two consecutive layers is weaker, and the forces that hold the layers together are the same forces that attract the individual crystals to each other and cause them to aggregate.

It is remarkable that in these minerals, there is no possibility to grow in the $\mathbf{c}$ direction by the addition of atoms or molecules in a classical crystal growth process. Both smectite and kaolinite have compound octahedral-tetrahedral layers (Figs $6 a$ and $b$ ) within which all changes are internally compensated, which prevents the addition of isolated ions in the c direction. Thus, the (001) faces parallel to the layers, formed by basal oxygen planes, are atomically flat, with no growth steps. The only possibility for growth in this direction is attachment of major units, i.e. oriented attachment of platelets comparable in lateral extent to the initial crystal. However, the $(h k 0)$ faces have $\mathrm{Si}-\mathrm{O}, M-\mathrm{O}$, and $M-\mathrm{OH}$ broken bonds, so they are rough (Fig. $6 a$ and $b$ ). Therefore, as shown in Fig. 7, these ( $h k 0)$ faces have growth steps, as in most silicates, and according to the classical growth theory the crystals can grow by atom-by-atom addition in the $\mathbf{a}$ and $\mathbf{b}$ directions. The weak attraction between two layers leads to their fusion on an energetically favourable process because it eliminates the two high-energysurfaces. After a nucleation stage, the laminar particles of these clay minerals can grow by atomby-atom addition in the [hk0] direction but only by $\mathrm{OA}$ in the [001] direction, resulting in anisotropically shaped particles only a few $\mathrm{nm}$ thick in the $\mathbf{c}$ direction.

Kaolinite particles are formed by the stacking of several $\mathrm{T}-\mathrm{O}$ layers in the [001] direction, and the aggregation is favoured by the opposite polar 


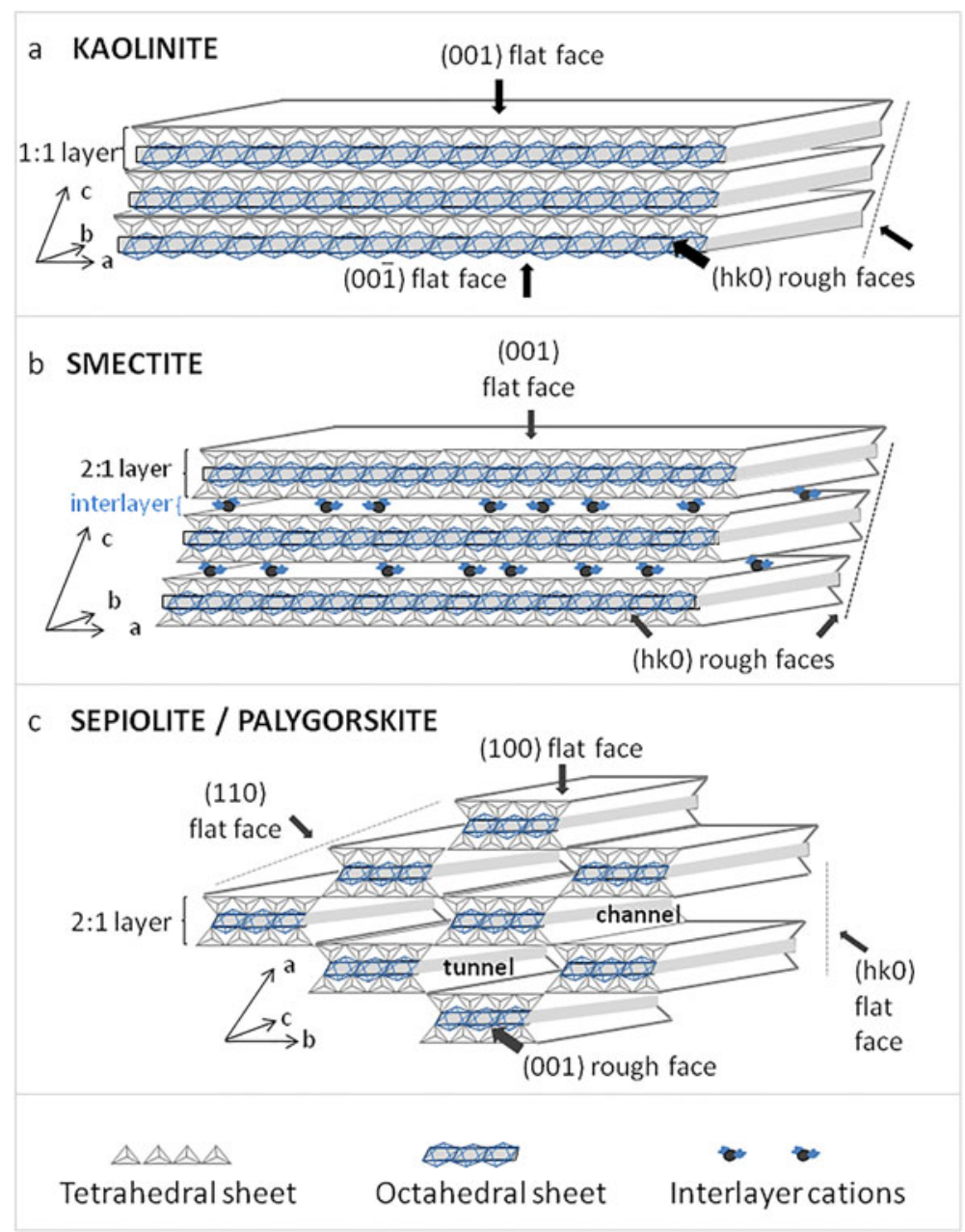

FIG. 6. Structural scheme of phyllosilicates: $(a)$ kaolinite (T-O); $(b)$ smectite (T-O-T); $(c)$ sepiolite/palygorskite (T-OT). Tetrahedral cations: $\mathrm{Si}^{4+}$, which can be replaced by $\mathrm{Al}^{3+}$. Octahedral cations: $\mathrm{Mg}, \mathrm{Al}, \mathrm{Fe}^{2+}$ or $\mathrm{Fe}^{3+}$ Interlayer cations: $\mathrm{Mg}^{2+}, \mathrm{Ca}^{2+}, \mathrm{Na}^{+}$or $\mathrm{K}^{+}$solvated by water.

charge of each sheet. The tetrahedral sheet has a negative polar charge, while the octahedral sheet has a positive polar charge; then when they aggregate, the tetrahedral sheet joins to the octahedral sheet only by elimination of the oriented water molecules at the interfaces, as previously described (Banfield et al., 2000) for iron oxyhydroxides. Likewise, smectite particles are formed by the stacking of several $\mathrm{T}-\mathrm{O}-\mathrm{T}$ layers. Unlike what occurs in kaolinite (Figs 1 and $8 b$ ), the smectite layers usually aggregate in a staggered manner (semi-oriented attachment) as described in De Yoreo et al. (2015), causing the particles to exhibit a sigmoidal cross-section (Figs 2, 3 and
$8 a$ ). The explanation for the staggered aggregation of layers must reside in the interlayer solvated cations. Additionally, staggering avoids the repulsive forces between two contiguous edges of layers because both are charged positively. The aggregation is easier when the position of the edge of a layer is near the face of the contiguous layer. In this manner, the positively charged edge of a layer is next to the negatively charged face of the next layer. In this configuration, positive charges of the broken octahedral bonds and the negative tetrahedral surface attract, which results in the sigmoidal shape of the particles. Smectite primary particles that aggregate are surrounded by hydrated cations. 


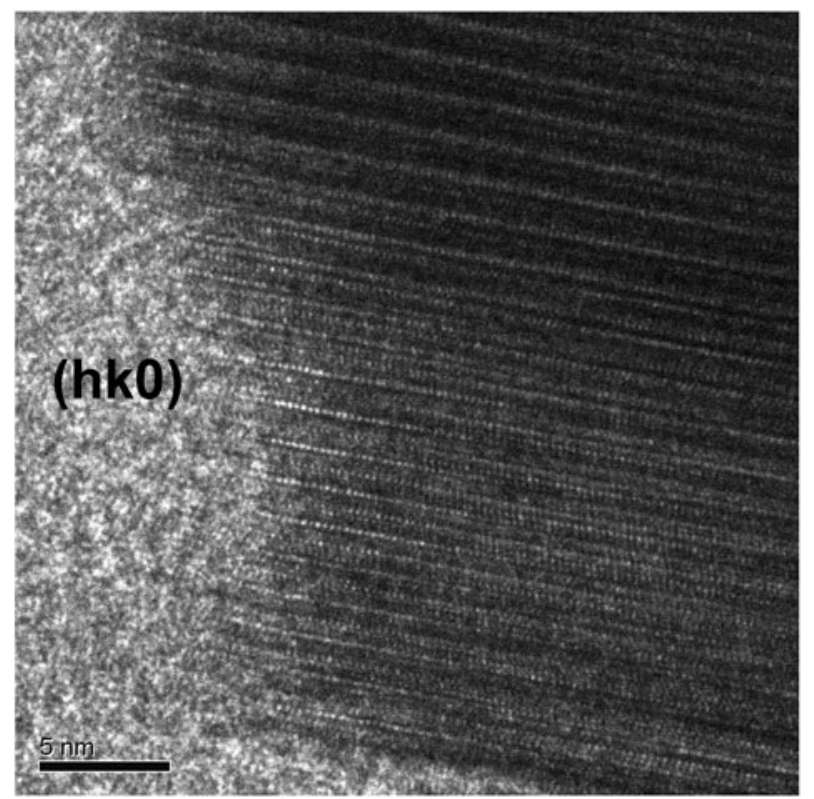

FIG. 7. Edge of smectite crystal, corresponding to a $(h k 0)$ face. It has numerous growth kinks, and a rough face with $\mathrm{Si}-\mathrm{O}$, $M-\mathrm{O}$, and $M-\mathrm{OH}$ bonds broken. In this face, the crystals can grow by atom-by-atom addition in this direction. The sample comes from The Pink Clays Unit, Tagus Basin, Spain.

For two layers to join, they need to expel half of the cations. Additionally, in contrast to what occurs in kaolinite, as a result of the aggregation with a small angle the smectite particles have a high number of stacking defects (edge dislocations) near the edge of the particles (Fig. 3). The defects appear to accommodate the structural differences between two particles. In the same way, small particles attach to each other to form larger particles. The aggregation phenomenon is responsible for the inclusion of the high number of defects, such as twists, disorientation, or tilts observed by HRTEM in clay minerals, notably in smectite (Figs 2 and 3).

In contrast, the $(h k 0)$ faces have a high number of kinks and very high attachment energy because they correspond to the edge of layers where both the tetrahedra and octahedra have broken bonds (Fig. 6). Therefore, in these faces, growth by atom-by-atom addition is possible. An image of the termination of the layers in a $(h k 0)$ face in a smectite is shown in Fig. 8. Although the energy decreases greatly when the addition of atoms completes the kinks, it returns (increases) to the previous level immediately after incorporation; thus, the crystal growth in this direction occurs by monomer addition.
Sepiolite and palygorskite are the end-members of a continuous polysomatic series (Suarez and García-Romero, 2013). In these fibrous clays, the apical oxygen atoms of the silica tetrahedral sheet are periodically inverted. This periodic inversion produces discontinuous octahedral sheets in the [010] direction, resulting in ribbons or polysomes along the [001] direction (Fig. 6c). Each ribbon is two silicate chains wide in palygorskite and three chains wide in sepiolite. Palygorskite contains Al and $\mathrm{Mg}$ as octahedral cations, while sepiolite contains only $\mathrm{Mg}$. The discontinuity in the octahedral sheet leads to the formation of tunnels at the inner and channels at the crystal edge, which run parallel to the $c$ axis of the crystal. In both minerals, $\mathrm{Mg}$ occupies the octahedral position located at the polysome edge and completes its coordination sphere with two water molecules. In the (001) faces, tetrahedral and octahedral sheets have broken bonds (Fig. 6c), and have high attachment energy (rough faces); therefore, the crystals can achieve growth by monomer addition, such as laminar clay minerals in the $(h k 0)$ directions. With this mechanism, the fibres can become longer but not wider because the $\mathbf{a}$ and $\mathbf{b}$ directions $(h k 0)$ lead to flat faces. In these flat faces, the atoms do not find kink growth. The $(h 00)$ faces 


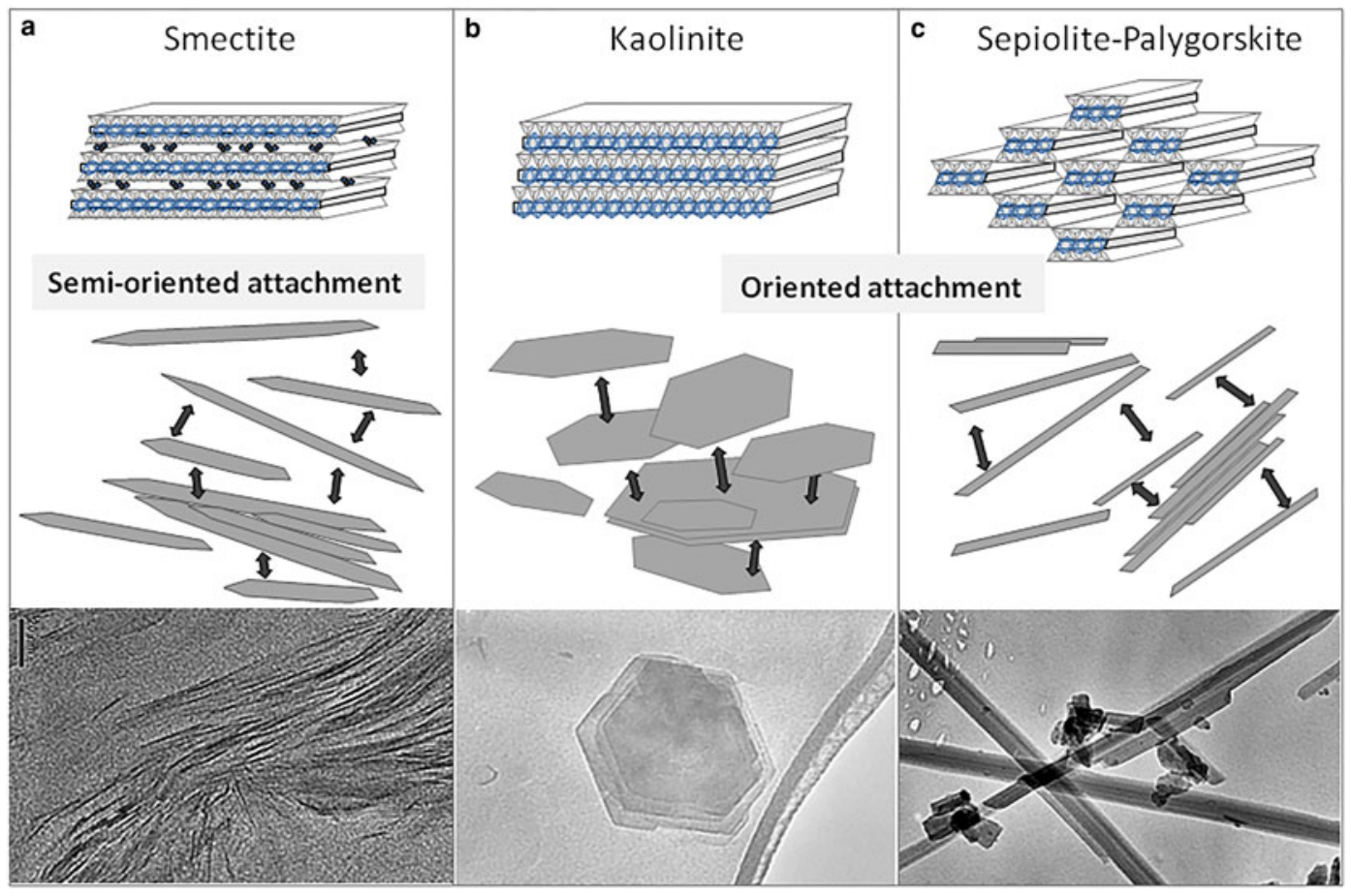

FIG. 8. Scheme of oriented aggregation in clay minerals. (a) Smectite. Particles with sigmoidal appearance are a consequence of their aggregation of several $\mathrm{T}-\mathrm{O}-\mathrm{T}$ layers in a staggered manner (semi-oriented attachment). Staggering avoids the repulsive forces between two contiguous edges of layer because both are charged positively, resulting in the sigmoidal shape of particles. (b) Kaolinite. Stacking of T-O layers in [001] direction. The aggregation will be favoured by the opposite polar charge of each sheet. (c) Sepiolite/palygorskite. The apical oxygens of the silica tetrahedral sheet are periodically inverted, producing discontinuous octahedral sheets in the $[0 k 0]$ direction, and resulting in ribbons or polysomes along the [001] direction, as well as tunnels and channels.

have the basal oxygen planes and finish in the channel, and the same occurs with the $(0 k 0)$ faces in which octahedral sheets finish with terminal octahedral cations bonded to water molecules. In these directions, it is impossible to achieve atomic growth by addition, while the only possibility for growth on the $(h k 0)$ faces is by the addition of complete units, such as in laminar clay minerals. However, in these cases, the units are the polysomes (or laths as larger units). The growth in the $(h k 0)$ directions is therefore by an oriented aggregation process, as shown in Fig. 8c. The oriented attachment of polysomes occurs along the fibre through the $\mathrm{Si}-\mathrm{O}-\mathrm{Si}$ bonds of alternating polysomes by an oxalation process in which a water molecule forms.

To summarize, the morphology of the resulting clay mineral crystals, both laminar (kaolinite and smectite) and fibrous (sepiolite-palygorskite), is controlled by the strong variation in attachment energy between different faces. The existence of crystal surfaces without broken bonds, and as a consequence weak attachment energy (001) in laminar and $(h k 0)$ in fibrous clay minerals, explains crystal growth by oriented aggregation. Smectites aggregate by semi-oriented attachment (Fig. 8a), while kaolinite, sepiolite and palygorskite aggregate by oriented attachment (Fig. $8 b$ and $c$ ). Growth by the atom-to-atom addition is possible only in the directions containing broken bonds, and consequently high attachment energy: the $(h k 0)$ faces in laminar phyllosilicates and the (001) faces in fibrous ones.

\section{Acknowledgements}

The authors acknowledge Dr. Lurdes Fernandez-Díaz and Dr. Cristobal Viedma for their valuable comments and the MINECO CGL2016-77005R project for providing financial support. 


\section{References}

Atkins, A., Shaw, S. and Peacock, C.L. (2014) Nucleation and growth of todorokite from birnessite: Implications for trace-metal cycling in marine sediments. Geochimica et Cosmochimica Acta, 144, 109-125.

Banfield, J.F., Welch, S.A., Zhang, H., Ebert, T.T., Penn, R.L. (2000) Aggregation-based crystal growth and microstructure development in natural iron oxyhydroxide biomineralization products. Science, 289, 751-754.

Colfen, H. and Antonietti, M. (2008) Mesocrystals and Nonclassical Crystallization. John Wiley and Sons Ltd., UK.

Cozzoli, P.D., Curri, M.L., Agostiano, A., Leo, G. and Lomascolo, M.J. (2003) ZnO nanocrystals by a nonhydrolytic route: synthesis and characterization. Journal of Physical Chemistry B, 107(20), 4756-4762.

De Yoreo, J.J., Gilbert, P.U.P.A., Som-merdijk, N.A.J.M., Penn, R.L., Whitelam, S., Joester, D., Zhang, H., Rimer, J.D., Navrot-sky, A., Banfield, J.F., Wallace, A. F., Michel, F.M., Meldrum, F.C., Cölfen, H. and Dove, P.M. (2015) Crystallization by particle attachment in synthetic, biogenic, and geologic environments. Science, 349, 6247.

García-Romero, E. and Suárez, M. (2014) Sepiolitepalygorskite polysomatic series: Oriented aggregation as a crystal growth mechanism in natural environments. American Mineralogist, 99, 1653-1661.

Helmy, H.M., Ballhaus, Ch., Fonseca, R.O.C., Wirth, R., Nagel, T. and Tredoux, M. (2013) Noble metal nanoclusters and nanoparticles precede mineral formation in magmatic sulphide melts. Nature Communications, 4, 2405.

Ivanov, V.K., Fedorov, P.P., Ye. Baranchikov, A. and Osiko, V.V. (2014) Oriented attachment of particles: 100 years of investigations of non-classical crystal growth. Russian Chemical Reviews, 83(12), 1204-1222.

Junge, M., Richard, W., Oberthür, T., Melcher, F. and Schreiber, A. (2015) Mineralogical siting of platinumgroup elements in pentlandite from the Bushveld Complex, South Africa. Mineralium Deposita, 50, 41-54.

Li, D., Nielsen, M.H., Lee, J.R.I., Frandsen, C., Banfield, J.F. and De Yoreo, J.J. (2012) Directionspecific interactions control crystal growth by oriented attachment. Science, 336, 1014-1018.
Niederberger, M., Krumeich, F., Hegetschweiler, K. and Nesper, R. (2002) An iron polyolate complex as a precursor for the controlled synthesis of monodispersed iron oxide colloids. Chemistry of Materials, 14, 78-82.

Nikolakis, V., Kokkoli, E., Tirrell, M., Tsapatsis, M. and Vlachos, D.G. (2000) Zeolite growth by addition of subcolloidal particles: Modeling and experimental validation. Chemistry of Matterials, 12, 845-853.

Penn, R.L. (2004) Kinetics of oriented aggregation. Journal of Physical Chemistry B, 108, 12707-12712.

Penn, R.L. and Banfield, J.F. (1998) Oriented attachment and growth, twinning, polytypism, and formation of metastable phases: Insights from nanocrystalline $\mathrm{TiO}_{2}$. American Mineralogist, 83, 1077-1082.

Penn, R.L. and Banfield, J.F. (1999) Morphology development and crystal growth in nanocrystalline aggregates under hydrothermal conditions: Insights from titania. Geochimica et Cosmochismica Acta, 63, 1549-1557.

Penn, R.L., Oskam, G., Strathmann, T.J., Searson, P.C., Stone, A.T. and Veblen, D.R. (2001) Epitaxial assembly in aged colloids. Journal of Physical Chemistry B, 105, 2177-2182.

Ricolleau, C., Audinet, L., Gandais, M. and Gacoin, T. (1999) Structural transformations in II-VI semiconductor nanocrystals. The European Physical Journal $D, 9,565-570$.

Suarez, M. and García-Romero, E. (2013) Sepiolitepalygorskite: a continuous polysomatic series. Clays and Clay Minerals, 61, 461-472.

Tessier, D. (1984) Etude de l'organisation des matériaux argileux. Hydration, gonflement et structuration au cours de la dessiccation et de la rehumectation. Thèse de Docteur ès Sciences de 1'Unive. Paris VII, France.

Tessier, D. and Pedro, G. (1987) Mineralogical characterization of 2:1 clays in soils: Importance of the clay texture. Pp. 78-84 in: Proceedings of the International Clay Conference, Denver (S.G. Shultz, H. van Olphen and F.A. Mumpton, editors). The Clay Minerals Society, Bloomington, USA.

Viedma, C., McBride, M. and Kahr, B. (2013) Enantiomer-specific oriented attachment: formation of macroscopic homochiral crystal aggregates from a racemic system. Angewandte Chemie International Edition, 52, 10545-10548.

Zhang, J.Z. (2000) Interfacial charge carrier dynamics of colloidal semiconductor nanoparticles. Journal of Physical Chemistry B, 104, 7239-7253. 